\title{
ON THE BOUNDARY CONDITION FOR THE LEGENDRE POLYNOMIALS
}

\author{
ÅKE PLEIJEL
}

\section{Introduction}

After a translation of the eigenvalue parameter, Legendre's differential equation is written $S u=\lambda T u$, where

$$
S=D\left(1-x^{2}\right) D+1, \quad T=1,
$$

and $D=i d / d x$. The equation is of Weyl's limit-circle type over the interval $I=\{x:-1<x<1\}$ so that for any non-real value $\lambda$ all solutions belong to $L^{2}(I)$. According to Weyl's theory a symmetric boundary condition must therefore be added to the differential equation in order to define a selfadjoint operator in $L^{2}(I)$. The symmetric boundary condition under which the Legendre polynomials are the eigenfunctions is given in Titchmarsh [3], p. 76-78, and is elaborated in Akhiezer-Glazman [1], p. $206-210$.

The author has generalized Weyl's theory to pairs of differential expressions

$$
S=\sum_{j=0}^{m} \sum_{k=0}^{m} D^{j} a_{j k} D^{k}, \quad T=\sum_{j=0}^{n} \sum_{k=0}^{n} D^{j} b_{j k} D^{k}
$$

on an interval $I$ of arbitrary type. The coefficients $a_{j k}$ and $b_{j k}$ shall be hermitean, $\bar{a}_{j k}=a_{k j}, \bar{b}_{j k}=b_{k j}$, and $S$ shall have a definite order $M$ on $I$ which is greater than the order of $T$. Dirichlet integrals

$$
\begin{aligned}
& J_{J}(u, v)^{T}=\int_{J} \sum_{j=0}^{n} \sum_{k=0}^{n} b_{j k} D^{k} u \overline{D^{j} v} d x, \\
& J_{J}(u, v)^{S}=\int_{J} \sum_{j=0}^{m} \sum_{k=0}^{m} a_{j k} D^{k} u \overline{D^{j} v} d x,
\end{aligned}
$$


are considered over compact subintervals $J$ of $I$. The more direct generalization of Weyl's theory is obtained under the condition that ${ }_{J}(u, u)^{T}$ is positive definite. Parallel to this $T$-positive theory there is a similar $S$-positive in which instead ${ }_{J}(u, u)^{S}$ is positive definite.

For the expressions (1.1) both Dirichlet integrals are positive definite so that in addition to the classical theory also an $S$-positive theory is available for Legendre's equation. But in this theory the equation is of limit-point type over $I=\{x:-1<x<1\}$, and Legendre's polynomials appear as eigenfunctions without any boundary condition.

The aim of this paper is to elucidate how the $S$-positive theory determines the symmetric boundary condition in the $T$-positive theory under which the Legendre polynomials are the eigenfunctions. In studying this question we shall consider expressions

$$
S=D p D+q \text { and } T=r
$$

on an open finite or infinite interval $I=\{x: a<x<b\}$. Here $p, q, r$ are sufficiently regular real functions which satisfy $p(x)>0$ and $0<r(x) \leqq C q(x)$ a.e. on $I$ with a positive constant $C$. Then the Dirichlet integrals

$$
\begin{aligned}
& { }_{J}(u, v)^{T}=\int_{J} r u \bar{v} d x, \\
& J_{J}^{(u, v)^{S}}=\int_{J}(p D u \overline{D v}+q u \bar{v}) d x
\end{aligned}
$$

are positive definite, and

$$
{ }_{j}(u, u)^{T} \leqq C_{J}(u, u)^{S} .
$$

The expressions (1.3) are real so that $\overline{S u}=S \bar{u}, \overline{T u}=T \bar{u}$. It is finally assumed that $S u=\lambda T u$ is in the limit-circle case over $I$ in the $T$ positive theory, while it is in the limit-point case over $I$ in the $S$-positive theory (explanation in Section 4).

The study relies upon general features of the $T$-positive and $S$-positive theories which are briefly accounted for in Sections 2-5. Generalizations to more complicated higher order equations $S u=\lambda T u$ are accessible although not deliberated in the text.

\section{Fundamentals of the theories for pairs of differential expressions}

The $T$ - and $S$-positive theories are founded on two Green's formulae on a linear space of ordered pairs $U=(u, u)$ of functions $u$ and $u$ 
satisfying $S u=T \dot{u}$, i.e. on a linear relation $S u=T \dot{u}$. It is assumed that $u$ belongs to the class $A(I)$ of complex valued functions $u$ with locally absolutely continuous first order derivatives. One can let $\dot{u}$ belong to the same class $A(I)$ so that the linear relation is

$$
E(I)=\{U=(u, \dot{u}) \in A(I) \times A(I): S u=T \dot{u}\} .
$$

The Green's formulae are

$$
\begin{aligned}
& i^{-1}\left({ }_{J}(\dot{u}, v)^{T}-{ }_{J}(u, \dot{v})^{T}\right)={ }_{J}\left[q_{z}^{T}(U, V)\right], \\
& i^{-1}\left({ }_{J}(\dot{u}, v)^{S}-{ }_{J}(u, \dot{v})^{S}\right)={ }_{J}\left[q_{z}^{S}(U, V)\right],
\end{aligned}
$$

where in the out-integrated parts

$$
\begin{aligned}
& q_{z}^{T}(U, V)=p D u \bar{v}+u \overline{p D v} \\
& q_{z}^{S}(U, V)=p D u \bar{v}+u \overline{p D v}
\end{aligned}
$$

at points $z$ in $I$. The formulae are valid when $U=(u, u)$ and $V=(v, \dot{v})$ belong to $\mathbb{E}(I)$. The first one is the usual Green's or Lagrangean formula. For more general expressions (1.2) similar formulae hold true with more complicated hermitean forms $q_{z}^{T}$ and $q_{z}^{S}$.

If the Dirichlet integral in question is positive, the finite or infinite limits

$$
\begin{aligned}
& { }_{I}(u, u)^{T}=\lim _{J \rightarrow I}(u, u)^{T} \leqq \infty, \\
& { }_{I}(u, u)^{S}=\lim _{J \rightarrow I}(u, u)^{S} \leqq \infty,
\end{aligned}
$$

exist when $u$ belongs to $A(I)$. By square brackets, []$^{T}$ or []$^{S}$, subspaces are indicated in which

$$
{ }_{I}(u, u)^{T}<\infty \quad \text { or } \quad{ }_{I}(u, u)^{S}<\infty
$$

remain finite for the contained functions. In this way $A[I]^{T}, A[I]^{S}$ are defined as linear subspaces of $A(I)$, and

$$
\begin{aligned}
& E[I]^{T}=\left\{U=(u, \dot{u}) \in A[I]^{T} \times A[I]^{T}: S u=T \dot{u}\right\}, \\
& E[I]^{S}=\left\{U=(u, \dot{u}) \in A[I]^{S} \times A[I]^{S}: S u=T \dot{u}\right\}
\end{aligned}
$$

in the $T$-positive and $S$-positive theories respectively. If

$$
{ }_{J} Q^{T}={ }_{J}\left[q_{z}^{T}\right], \quad{ }_{J} Q^{S}={ }_{J}\left[q_{z}^{S}\right]
$$

are the right hand sides of the Green's formulae (2.1), (2.2), one obtains by letting $J$ tend to $I$ that

$$
\begin{array}{ll}
{ }_{I} Q^{T}(U, V)=i^{-1}\left({ }_{I}(\dot{u}, v)^{T}-{ }_{I}(u, \dot{v})^{T}\right) & \text { on } E[I]^{T}, \\
{ }_{I} Q^{S}(U, V)=i^{-1}\left({ }_{I}(u, v)^{S}-{ }_{I}(u, \dot{v})^{S}\right) & \text { on } E[I]^{S} .
\end{array}
$$


Here

$$
{ }_{I} Q^{T}=q_{b}^{T}-q_{a}^{T}, \quad{ }_{I} Q^{S}=q_{b}^{S}-q_{a}^{S},
$$

where $q_{b}^{T}, q_{a}^{T}$ and $q_{b}^{S}, q_{a}^{S}$ are the finite limits of $q_{z}^{T}$ and $q_{z}^{S}$ on $E[I]^{T}$ and $E[I]^{S}$.

The dimension of a solution space

$$
E_{\lambda}(I)=\{U=(u, \lambda u): u \text { regular, } S u=\lambda T u\}
$$

equals the order $M$ of $S u=\lambda T u$ i.e. is $M=2$ for $S$ and $T$ in (1.3). The square bracket solution spaces are

$$
\begin{aligned}
& E_{\lambda}[I]^{T}=\left\{U=(u, \lambda u) \in E_{\lambda}(I):{ }_{I}(u, u)^{T}<\infty\right\}, \\
& E_{\lambda}[I]^{S}=\left\{U=(u, \lambda u) \in E_{\lambda}(I):{ }_{I}(u, u)^{S}<\infty\right\}
\end{aligned}
$$

in a $T$-positive or $S$-positive theory. As subspaces of $E_{\lambda}(I)$ they are at most 2-dimensional ( $M$-dimensional) but can have lower dimensions including the dimension 0 . Putting $\dot{u}=\lambda u, \dot{v}=\lambda v$ in (2.5), (2.6) when $U, V$ belong to $E_{\lambda}[I]^{T}$ or $E_{\lambda}[I]^{S}$, one obtains

$$
\begin{array}{ll}
{ }_{I} Q^{T}(U, V)=c(\lambda)_{I}(u, v)^{T} & \text { on } E_{\lambda}[I]^{T}, \\
{ }_{I} Q^{S}(U, V)=c(\lambda)_{I}(u, v)^{S} & \text { on } E_{\lambda}[I]^{S}
\end{array}
$$

with $c(\lambda)=i^{-1}(\lambda-\bar{\lambda})$. Hence, $c(\lambda)_{I} Q^{T}$ and $c(\lambda){ }_{J} Q^{S}$ are positive definite on $E_{\lambda}[I]^{T}$ and on $E_{\lambda}[I]^{S}$ respectively. Similar formulae and positivity statements are evidently valid for $c(\lambda){ }_{J} Q^{T}, c(\lambda){ }_{J} Q^{S}$ on $E_{\lambda}(I)$ when $J$ is a compact subinterval of $I$, and the Dirichlet integrals ${ }_{J}\left(\cdot^{\cdot},\right)^{T}$ or ${ }_{J}(\cdot, \cdot)^{S}$ are positive definite.

The T-positive and S-positive theories for the pair S, T are essentially the theories for the hermitean forms ${ }_{I} Q^{T}$ on $E[I]^{T}$ and ${ }_{I} Q^{S}$ on $E[I]^{S}$. After definition of symmetric boundary conditions in $E[I]^{T}$ and $E[I]^{S}$, these theories lead to spectral theorems on the completions $\overline{A[I]^{T}}$ or $\overline{A[I]^{S}}$ with respect to the norm defined by the positive Dirichlet integral taken over $I$. When $S, T$ are given by (1.3) the space $\overline{A[I]^{T}}$ is the Hilbert space of functions which are integrable square over $I$ with the weight function $r$. Under the condition (1.6) the Hilbert space $\overline{A[I]^{S}}$ has another scalar product but is a subset of the previous one.

Even if not strictly necessary for the theories above it is, however, natural and advantageous to consider pairs $U=(u, \dot{u})$ of functions $u, \dot{u}$ only defined and regular on intervals

$$
I_{a}=\{x: a<x \leqq c-0\}, \quad I_{b}=\{x: c+0 \leqq x<b\} .
$$

More generally the intervals $I_{a}, I_{b}$ are taken adjacent to one endpoint of $I$ excluding the other one. The previous definitions are adapted when 
$I$ is replaced by the interrupted interval $\hat{I}=I_{a} \cup I_{b}$. In this way $A(\hat{I})$ and $E(\hat{I}), E[\hat{I}]^{T}, E[\hat{I}]^{S}, E_{\lambda}(\hat{I}), E_{\lambda}[\hat{I}]^{T}, E_{\lambda}[\hat{I}]^{S}$ are defined. The space $E[\hat{I}]^{T}$ in a $T$-positive theory is for instance cut out from $E(\hat{I})$ by the conditions for $U=(u, \dot{u})$ that the integrals $\hat{I}(u, u)^{T}<\infty, \hat{I}(\dot{u}, \dot{u})^{T}<\infty$ over $\hat{I}=I_{a} \cup I_{b}$ shall be finite. The hermitean forms

$$
\begin{aligned}
& { }_{J} Q^{T}(U, V)=q_{y}^{T}(U, V)-q_{x}^{T}(U, V), \\
& { }_{J} Q^{S}(U, V)=q_{y}^{S}(U, V)-q_{x}^{S}(U, V)
\end{aligned}
$$

are defined on $E(\hat{I})$ when the endpoints $x$ and $y$ of $J$ belong, one to $I_{a}$, the other one to $I_{b}$. The Green's formulae are of course only valid for arbitrary compact intervals $J$ when the functions in $U, V$ are defined and regular on the uninterrupted interval $I$. Otherwise the formulae can be replaced by

$$
{ }_{J^{\prime}} Q(U, V)-{ }_{J} Q(U, V)=i^{-1}\left(J_{J^{\prime}-J}(\dot{u}, v)-{ }_{J^{\prime}-J}(u, \dot{v})\right)
$$

with superscripts $T$ or $S$, and with the lower and upper endpoints of $J$ and $J^{\prime}, J \subset J^{\prime}$, lying in $I_{a}$ and $I_{b}$ respectively.

The spaces $E(I), E[I]^{T}$ etc. are considered as subspaces of $E\left(\hat{I)}, E[\hat{I}]^{T}\right.$ etc. Observe for instance that the dimension of $E_{\lambda}(\hat{I})$ is $2 M=4$, while the dimension of $E_{\lambda}(I)$ is $M=2$ for the expressions $S$ and $T$ in (1.3). Later on (Section 5) also notations such as $E\left[I_{b}\right]^{S}$ are used. In the spaces $E\left[I_{b}\right]^{S}, E\left[I_{b}\right]^{T}$ integrability conditions are imposed only at $x=b$ since $x=c+0$ is a "regular" point.

\section{Maximal regular subspaces and symmetric boundary conditions}

By extraction of the underlying ideas in Weyl's method with contracting circles it can be proved that for any non-real $\lambda$, the direct sums

$$
\begin{aligned}
& R_{\lambda}[I]^{T}=E_{\lambda}[I]^{T}+E_{\bar{\lambda}}[I]^{T}, \\
& R_{\lambda}[I]^{S}=E_{\lambda}[I]^{S}+E_{\bar{\lambda}}[I]^{S}
\end{aligned}
$$

are maximal regular subspaces of $E[\hat{I}]^{T}$ and of $E[\hat{I}]^{S}$ with respect to ${ }_{I} Q^{T}$ and ${ }_{I} Q^{S}$. The meaning of this will presently be explained for (general) $T$-positive and $S$-positive theories simultaneously, by observing that these theories are both concerned with an hermitean form $Q \quad\left({ }_{I} Q^{T}\right.$ or $\left.{ }_{I} Q^{S}\right)$ on a linear space $E\left(E[\hat{I}]^{T}\right.$ or $\left.E[\hat{I}]^{S}\right)$. The proof itself consists of a transition to the limit which transfers elementary properties of ${ }_{J} Q^{T}$ or ${ }_{J} Q^{S}$ on $E(\hat{I})$ and $E_{\lambda}(I)$ into the statements about (3.1), (3.2). 
A finite dimensional subspace $R$ of $E$ is regular with respect to $Q$ if $Q$ is non-degenerate or "regular" on $R$. If $(p, n)$ is the signature of $Q$ on $R$ i.e. if $(p, n)$ is the pair of the positive and negative inertia indices of $Q$ on $R$, then the regularity of $R$ is equivalent to $\operatorname{dim} R=p+n$. The space $R$ is maximal regular if for any $U$ in $E$ but outside $R$, the linear hull $\{U, R\}$ is not regular. Every $U$ in $E$ has a unique $Q$-projection $U^{\prime}$ on a regular space $R$ determined by $U^{\prime} \in R, Q\left(U-U^{\prime}, R\right)=0$ so that $Q\left(U-U^{\prime}, V\right)=0$ for all $V$ in $R$. If $R$ is maximal regular, $Q\left(U-U^{\prime}, E\right)=0$. In this case

$$
Q(U, V)=Q\left(U^{\prime}, V^{\prime}\right)
$$

for any two elements $U, V$ in $E$ and their projections $U^{\prime}, V^{\prime}$ on $R$. The theory of the values of $Q$ on $E$ is in this way reduced to a study of the values on a finite dimensional space $R$. All maximal regular subspaces $R$ (if they exist) are isomorphic, and $Q$ has the same signature $(p, n)$ on all of them.

The statements about (3.1), (3.2) tell that maximal regular subspaces exist for ${ }_{I} Q^{T}$ on $E[\hat{I}]^{T}$ and for ${ }_{I} Q^{S}$ on $E[\hat{I}]^{S}$. The spaces $R_{\lambda}[I]^{T}, R_{\lambda}[I]^{S}$ are maximal regular for all non-real values of $\lambda$. According to (2.7), (2.8) the signatures of $c(\lambda){ }_{I} Q^{T}$ and $c(\lambda){ }_{I} Q^{S}$ on these spaces are $\left(\operatorname{dim} E_{\lambda}[I]^{T}, \operatorname{dim} E_{\bar{\lambda}}[I]^{T}\right)$ and $\left(\operatorname{dim} E_{\lambda}[I]^{S}, \operatorname{dim} E_{\bar{\lambda}}[I]^{S}\right)$. An important consequence is that $\operatorname{dim} E_{\lambda}[I]^{T}$ and $\operatorname{dim} E_{\lambda}[I]^{S}$ are independent of $\lambda$ in the half-planes $\operatorname{Im}(\lambda)>0$ and $\operatorname{Im}(\lambda)<0$. In general there exist other maximal regular subspaces than (3.1), (3.2) in $E[\hat{I}]^{T}$ and $E[\hat{I}]^{S}$, also such which are not subspaces of $E[I]^{T}$ and $E[I]^{S}$ as (3.1), (3.2) are.

A linear subspace $Z$ of $E$ is a $Q$-nullspace if $Q(Z, Z)=0$ i.e. if $Q(U, V)=0$ for all $U, V$ in $Z$. The $Q$-projection $Z_{R}^{\prime}$ of a $Q$-nullspace $Z$ into a maximal regular subspace $R$ is a $Q$-nullspace in $R$ since $Q\left(U^{\prime}, V^{\prime}\right)=Q(U, V)$ under such projections. If for one $R$ the space $Z_{R}^{\prime}$, as a $Q$-nullspace in $R$ is maximal, i.e. if according to Sylvester's inertia theorem

$$
\operatorname{dim} Z_{R}^{\prime}=\min (p, n),
$$

then the same is true for any maximal regular subspace. A nullspace $Z$ is always contained in $E^{\perp}+Z_{R}^{\prime}$, where

$$
E^{\perp}=\{U \in E: Q(U, E)=0\},
$$

and $\mathbb{E}^{\perp} \dot{+} Z_{R}^{\prime}$ is again a $Q$-nullspace. Under the condition (3.3) the space $E^{\perp} \mp Z_{R}^{\prime}$ does not allow any proper extension to a larger $Q$-nullspace in $E$. The extension $E^{\perp} \dot{+} Z_{R}^{\prime}$ is then the same for all choices of the maximal regular $R$ and is called a symmetric boundary condition in $E$. The symmetric boundary condition is uniquely determined by a $Z$ which satisfies 
(3.3). Because of this uniqueness, already $Z$ may be called a symmetric boundary condition. This in particular applies to a $Q$-nullspace $Z^{\prime}$ in a maximal regular subspace $R$ provided $\operatorname{dim} Z^{\prime}$ equals $\min (p, n)$. In $T$-positive or $S$-positive theories for a pair $S, T$ of differential expressions there exist maximal regular subspaces already in $E[I]^{T}$ and $E[I]^{S}$, namely (3.1), (3.2). Therefore any symmetric boundary condition can be defined in $E[I]^{T}$ or $E[I]^{S}$ and then has a unique extension to one in $E[\hat{I}]^{T}$ or $E[\hat{I}]^{S}$.

A sufficient condition for (3.3) is that

$$
\operatorname{dim} Z \cap R=\min (p, n)
$$

for one choice of a maximal regular subspace $R$. For, $Z_{R}^{\prime} \supset Z \cap R$ and $Z \cap R$ is a $Q$-nullspace in $R$.

If a nullspace $Z$ satisfies (3.3) and coincides with its maximal extension to a $Q$-nullspace in $E$, then $Z_{R}^{\prime}$ and $Z \cap R$ are the same for all maximal regular subspaces $R$.

Observe that a symmetric boundary condition in theories for differential expressions $S, T$ is a condition for a pair $(u, u)$ which is natural since $q_{z}^{T}(U, V)$ and $q_{z}^{S}(U, V)$ contain $\dot{u}$ and $\dot{v}$. Only exceptionally, when $T$ means multiplication by a function as in (1.3), the form $q_{z}^{T}(U, V)$ does not contain $\dot{u}$ and $\dot{v}$ so that symmetric boundary conditions may be formulated in the classical way.

\section{Limit types over an interval}

The limit type over the interval $I$ of a pair $S, T$ in a $T$-positive theory is the signature $(p, n)$ of ${ }_{I} Q^{T}$ on a maximal regular subspace of $E[\hat{I}]^{T}$. According to (2.7) and the statement about (3.1) this limit type over $I$ is

$$
\begin{array}{ll}
p=\operatorname{dim} E_{\lambda}[I]^{T} & \text { for } \operatorname{Im}(\lambda)>0, \\
n=\operatorname{dim} E_{\bar{\lambda}}[I]^{T} & \text { for } \operatorname{Im}(\lambda)>0 .
\end{array}
$$

Similarly the limit type over $I$ in a $S$-positive theory is the pair $(p, n)$ of numbers

$$
\begin{array}{ll}
p=\operatorname{dim} E_{\lambda}[I]^{S} & \text { for } \operatorname{Im}(\lambda)>0, \\
n=\operatorname{dim} E_{\bar{\lambda}}[I]^{S} & \text { for } \operatorname{Im}(\lambda)>0 .
\end{array}
$$

The pairs in (4.1) and (4.2) evidently satisfy

$$
(0,0) \leqq(p, n) \leqq(M, M),
$$

with $M=2$ for a second order equation $S u=\lambda T u$. 
The pair $S, T$ is of limit-point type over $I$ in the $S$-positive theory for instance, if for (4.2) equality holds to the left in (4.3) i.e. if $\operatorname{dim} E_{\lambda}[I]^{S}=0$ for all non-real values of $\lambda$. This means that for any non-real $\lambda$ the only solution of $S u=\lambda T u$ for which ${ }_{I}(u, u)^{S}<\infty$, is the trivial one $u$ identically 0 . If $\operatorname{dim} E_{\lambda}[I]^{S}=0$ for all non-real $\lambda$, then $R_{\lambda}[I]^{S}=\{0\}$. Since ${ }_{I} Q^{S}(U, V)={ }_{I} Q^{S}\left(U^{\prime}, V^{\prime}\right)$ under $Q$-projections on a maximal regular space, the form ${ }_{I} Q^{S}(U, V)$ vanishes for all $U, V$ in $E[\hat{I}]^{S}$ so that

$$
{ }_{I} Q^{S}(U, V)=\lim _{J \rightarrow I} Q^{S}(U, V)=0 \quad \text { on } E[\hat{I}]^{S}
$$

in the limit-point case over $I$.

Because of (2.8) the vanishing of ${ }_{I} Q^{S}$ conversely implies that $E_{\lambda}[I]^{S}=\{0\}$ for $\lambda$ non-real.

In the other extreme case with equality on the right hand side of (4.3), the pair $S, T$ is of limit-circle type over $I$. In the $T$-positive theory for instance, this means that $E_{\lambda}[I]^{T}$ has the same dimension $M \quad(M=2$ for a second order equation) as $E_{\lambda}(I)$ so that $E_{\lambda}[I]^{T}=E_{\lambda}(I)$ for all nonreal values of $\lambda$. This is equivalent to the statement that in the limit-circle case over $I,{ }_{I}(u, u)^{T}<\infty$ for all regular solutions of $S u=\lambda T u$ when $\lambda$ is non-real. Between the limit-point and limit-circle cases there is in general a sequence of intermediate possibilities.

\section{Limit types at the endpoints}

For a detailed study of ${ }_{I} Q^{T}=q_{b}^{T}-q_{a}^{T}$ on $E[\hat{I}]^{T}$ it is necessary to investigate the hermitean forms $q_{b}^{T}$ and $q_{a}^{T}$ on $E\left[I_{b}\right]^{T}$ and $E\left[I_{a}\right]^{T}$, see end of Section 2. Similarly for ${ }_{I} Q^{S}=q_{b}^{S}-q_{a}^{S}$. For the differential expressions (1.3) the study of $q_{b}^{T}$ follows Weyl's original method applied to $I_{b}=$ $\{x: c+0 \leqq x<b\}$ instead of $\{x: 0 \leqq x<\infty\}$, and the study of $q_{a}^{T}$ is done in a similar way. For more general expressions $S$ (Everitt, Kodaira, Kimura-Takahasi) and for two general expressions $S$ and $T$ in a $T$-positive or $S$-positive theory (Pleijel) generalizations are needed. If the order of $S$ is even, $M=2 \mathrm{~m}$, the result of such a study can be concentrated in the formulae

$$
\begin{aligned}
& \operatorname{dim} E_{\lambda}\left[I_{a}\right] \geqq m, \quad \operatorname{dim} E_{\lambda}\left[I_{b}\right] \geqq m, \\
& \operatorname{dim} E_{\lambda}\left[I_{a} \cup I_{b}\right]=\operatorname{dim} E_{\lambda}[I]+M,
\end{aligned}
$$

which are valid with superscripts $T$ or $S$ when $\lambda$ is non-real. If $S$ is of odd order in a $T$-positive theory, the right hand sides of the formulae (5.1) are slightly modified. Clearly $\operatorname{dim} E_{\lambda}\left[I_{a} \cup I_{b}\right]=\operatorname{dim} E_{\lambda}\left[I_{a}\right]+\operatorname{dim} E_{\lambda}\left[I_{b}\right]$. For the expressions (1.3) $M=2$ and $m=1$. 
Observe that for instance $\operatorname{dim} E_{\lambda}\left[I_{b}\right]^{S}$ is the number of linearly independent solutions of $S u=\lambda T u$ for which ${ }^{b}(u, u)^{S}<\infty$ is finite when integrated over any interval adjacent to $b$, but excluding $a$, in particular over $I_{b}=\{x: c+0 \leqq x<b\}$. When the number above equals $m$, or is 1 for the expressions (1.3), the pair $S, T$ is of limit point type at the endpoint $x=b$ in an $S$-positive theory. A consequence of $(5.1),(5.2)$ is

$\mathrm{R}$ e $\mathrm{m}$ a $\mathrm{rk}$ 5.1. The pair $S, T$ is of limit-point type over $I$ in an S-positive theory if and only if it is of limit-point type at both endpoints a and $b$. In the limit-point case $\operatorname{dim} E_{\lambda}[\hat{I}]^{S}=M$ which for the expressions (1.3) is $M=2$.

A corresponding remark holds true in a $T$-positive theory and is well known when $T=1$.

The limit-circle case at an endpoint, $b$ for instance, occurs when all solutions of $S u=\lambda T u$ give finite values to $I_{b}(u, u)^{T}$ or ${ }_{I_{b}}(u, u)^{S}$ respectively, so that $\operatorname{dim} E_{\lambda}\left[I_{b}\right]^{T}=M$ or $\operatorname{dim} E_{\lambda}\left[I_{b}\right]^{S}=M$. The pair $S, T$ is of limit-circle type over $I$ if and only if it is of limit-circle type at both endpoints $a$ and $b$.

Interesting for our purposes is that according to (5.2) $\operatorname{dim} E_{\lambda}[\hat{I}] \geqq$ $\operatorname{dim}\left(E_{\lambda}[I]+E_{\bar{\lambda}}[I]\right)$ with superscripts $T$ or $S$. This is an immediate consequence of $M \geqq \operatorname{dim} E_{\bar{\lambda}}[I]$. Hence $E_{\lambda}[\hat{I}]$ can be maximal regular only if $\operatorname{dim} E_{\bar{\lambda}}^{-}[I]=M$. For reference we state

$\mathrm{R}$ e m a r k 5.2. For the expressions (1.3) the space $E_{\lambda}[\hat{I}]^{T}$ is actually a maximal regular subspace of $E[\hat{I}]^{T}$ in the limit-circle case over $I$ in a T-positive theory.

In [3], Chapter II, the solutions $\varphi$ and $\vartheta$ of $S u=\lambda T u$ give a base $\Phi=(\varphi, \lambda \varphi), \quad \Theta=(\vartheta, \lambda \vartheta)$ of $E_{\lambda}\left[I_{b}\right]^{T} \quad(x=0 \quad$ and $\quad x=\infty$ in [3] correspond to $x=c+0, x=b)$. Computation of $q_{b}^{T}(\Phi, \Phi), q_{b}^{T}(\Phi, \Theta)$ and $q_{b}^{T}(\Theta, \Theta)$ shows that in the limit-circle case $q_{b}^{T}$ has the signature $(1,1)$ on $E_{\lambda}\left[I_{b}\right]^{T}$, hence is regular on this 2-dimensional space. As a consequence ${ }_{I} Q^{T}=q_{b}^{T}-q_{a}^{T}$ is regular on $E_{\lambda}[\hat{I}]^{T}$. The discussion makes use of Weyl's criterion for the limit-circle case in terms of his formula for the radii of the contracting circles. This formula is valid for the real expressions (1.3). For general expressions (1.2) the corresponding discussion is more complicated.

\section{The original question. A nullspace}

Recall the assumption in Section 1 that the pair $S, T$ is of limitpoint type in an $S$-positive theory and that 


$$
0 \leqq{ }_{J}(u, u)^{T} \leqq C_{J}(u, u)^{S}
$$

with a positive constant $C$. The last inequality implies that $E[\hat{I}]^{S} \subset E[\hat{I}]^{T}$.

Consider $S^{\alpha}=S+\alpha T$ for a real positive value $\alpha$. By the definitions of the Dirichlet integrals, in the actual case (1.4) and (1.5), it follows that

$$
{ }_{J}(u, v)^{S^{\alpha}}={ }_{J}(u, v)^{S}+\alpha_{J}(u, v)^{T} .
$$

Hence also $S^{\alpha}$ has a positive definite Dirichlet integral. The equation $S u=\lambda T u$ can be written $S^{\alpha} u=(\lambda+\alpha) T u$, where the values $\lambda, \lambda+\alpha$ are non-real simultaneously. It follows that if the pair $S, T$ is of limitpoint type in the $S$-positive theory, the same holds true for $S^{\alpha}, T$ in an $S^{\alpha}$-positive theory. On account of $(6.1)$ it is easy to see that if $U=(u, \dot{u})$ belongs to $E[\hat{I}]^{S}$, then $U^{\alpha}=\left(u, \dot{u}^{\alpha}\right)$ with $\dot{u}^{\alpha}=\dot{u}+\alpha u$ belongs to $E[\hat{I}]^{S^{\alpha}}$. Let $U=(u, \dot{u}), V=(v, \dot{v})$ be elements of $E[\hat{I}]^{S}$ and put $U^{\alpha}=(u, \dot{u}+\alpha u), V^{\alpha}=(v, \dot{v}+\alpha v)$. According to (4.4) ${ }_{J} Q^{S}(U, V)$ and $Q^{S^{\alpha}}\left(U^{\alpha}, V^{\alpha}\right)$ tend to 0 when $J$ tends to $I$. The form ${ }_{J} Q^{S^{\alpha}}\left(U^{\alpha}, V^{\alpha}\right)$ equals ${ }_{j}\left[q_{z}^{S^{\alpha}}\left(U^{\alpha}, V^{\alpha}\right)\right]$, and a computation based upon (2.3), (2.4) shows that

$$
q_{z}^{S^{\alpha}}\left(U^{\alpha}, V^{\alpha}\right)=q_{z}^{S}(U, V)+\alpha q_{z}^{T}(U, V)
$$

so that

$$
{ }_{J} Q^{S^{\alpha}}\left(U^{\alpha}, V^{\alpha}\right)={ }_{J} Q^{S}(U, V)+\alpha_{J} Q^{T}(U, V) .
$$

Letting here $J$ tend to $I$ it follows from the previous statements that if $U$ and $V$ belong to $E[\hat{I}]^{S}$, then

$$
{ }_{I} Q^{T}(U, V)=\lim _{J \rightarrow I} Q^{T}(U, V)=0 .
$$

The subspace $E[\hat{I}]^{S}$ of $E[\hat{I}]^{T}$ is a nullspace for ${ }_{I} Q^{T}$.

This statement remains true also for higher order expressions $S$ and $T$ under the only condition (6.1) combined with the assumption that the pair $S, T$ is of limit-point type in the $S$-positive theory. As a matter of fact the identity (6.3) is valid also for the forms $q_{z}^{S}, q_{z}^{T}$ which appear in the theory of the expressions (1.2).

\section{The nullspace is a symmetric boundary condition}

To see that the nullspace $Z=E[\hat{I}]^{S}$ for ${ }_{I} Q^{T}$ is a symmetric boundary condition in $E[\hat{I}]^{T}$, it is sufficient to verify (3.4) for one maximal regular subspace $R$. According to Remark 5.2 we can take $R=E_{\lambda}[\hat{I}]^{T}$ when the 
pair $S, T$ in (1.3) is in the limit-circle case in the $T$-positive theory. With $Z=E[\hat{I}]^{S}$ the intersection $Z \cap E_{\lambda}[\hat{I}]^{T}$, where in the limit-circle case $E_{\lambda}[\hat{I}]^{T}=E_{\lambda}(\hat{I})$, equals $E_{\lambda}[\hat{I}]^{S}$. But according to Remark 5.1 the space $E_{\lambda}[\hat{I}]^{S}$ is 2-dimensional which is the right dimension in (3.4) since the limit type of $S, T$ in the $T$-positive theory is $(p, n)=(2,2)$. Thus the T-positive theory inherits $Z=E[\hat{I}]^{S}$ from the S-positive theory as a symmetric boundary condition. In concentration this is our final result.

\section{Concluding remarks}

The aim of the $T$ - and $S$-positive theories is to connect the pair $S, T$ with a Hilbert space $H$ in a useful way. In the $T$-positive theory for the expressions (1.3), $H=\overline{A[I]^{T}}$ is the function space with scalar product $\int_{a}^{b} r u \bar{v} d x$. Also for more general pairs (1.2) the Hilbert space is similarly determined before the introduction of symmetric boundary conditions. The signature $(p, n)$ of ${ }_{I} Q^{T}$ or ${ }_{I} Q^{S}$ on a maximal regular space serves as a pair of deficiency indices. Only when $p=n$ (as for the real operators (1.3)), there exist selfadjoint operators or relations on $H$ connected with the pair $S, T$. According to (2.5), (2.6) a symmetric boundary condition, i.e. a maximal nullspace for ${ }_{I} Q^{T}$ or ${ }_{I} Q^{S}$ considered in $E[I]^{T}$ or $E[I]^{S}$, is a symmetric relation

$$
{ }_{I}(\dot{u}, v)={ }_{I}(u, \dot{v})
$$

(superscript $T$ or $S$ ), and can be shown to have a selfadjoint extension in the Hilbert space if $p=n$. The essential feature of the spectral theorem for the selfadjoint extension is the expansion or representation of an arbitrary element $u$ of $H$ in terms of eigenfunctions, or in general in terms of $H$-projections on eigenspaces which can be arranged to belong to finite intervals of the spectral axis. The eigenfunctions and the elements of such eigenspaces can be proved to be regular functions $u$ for which $S u$ and $T u$ have their straight forward meaning. The simplest case is when the spectrum is discrete. According to a classical theorem, recently extended to general differential expressions (1.2) by Bert Karlsson, this is certainly the case when the pair $S, T$ is of limit-circle type. When the spectrum is discrete, a symmetric boundary condition together with $S u=\varrho T u$ ( $\varrho$ eigenvalue, real) characterizes the eigenfunctions i.e. the expansion terms. For the expressions (1.3) the condition that $U=(u, u)$ belongs to $Z=E[I]^{S}\left(\subset E[I]^{T}\right)$ is that ${ }_{I}(u, u)^{S}<\infty{ }_{I}(\dot{u}, \dot{u})^{S}<\infty$. In the actual case of an eigenfunction $u, \dot{u}=\varrho u$, the condition that $u$ satisfies $Z=E[I]^{S}$ reduces to the one condition that 


$$
\int\left(p|D u|^{2}+q|u|^{2}\right) d x
$$

is finite over neighbourhoods of $x=a$ and $x=b$. For the Legendre expressions (1.1) the condition that an element $u$ in $H=L^{2}(-1,1)$ also belongs to $Z=E[I]^{S}$ is that

$$
\int\left(1-x^{2}\right)\left|u^{\prime}\right|^{2} d x
$$

is finite over neighbourhoods of $x=-1$ and $x=1$. In this form the symmetric boundary condition inherited from the $S$-positive theory has since long been used by the physicists to select the Legendre polynomials as eigenfunctions. It is given in Akhiezer-Glazman's book.

Other equivalent forms of (8.1) can be deduced as in Akhiezer-Glazman [1] because of the simple form of $q_{z}^{S}$ when $M=2$. They are difficult to generalize to cases when $M>2$, and are at least for selection purposes of minor interest.

\title{
References
}

[1] Akhiezer, N. I., and I. M. Glazman: Theory of linear operators in Hilbert space. II. - [Second printing.] Frederick Ungar Publishing Co., New York, 1963.

[2] PleiJel, A.: A survey of spectral theory for pairs of ordinary differential operators. - Spectral theory and differential equations (Symposium, Dundee, 1974) edited by W. N. Everitt, Lecture Notes in Mathematics 448, Springer-Verlag, Berlin - Heidelberg - New York, 1975, 256-272.

[3] Titchmarsh, E. C.: Eigenfunction expansions associated with second-order differential equations. I. - [Second edition.] Clarendon Press, Oxford, 1962 .

\author{
Uppsala University \\ Department of Mathematics \\ Sysslomansgatan 8 \\ S-752 23 Uppsala \\ Sweden
}

Received 22 August 1975 